\title{
Effect of Temperature on the Refractive Indices of Liquid Crystals and Validation of a Modified Four-Parameter Model
}

\author{
K.D. Thingujam ${ }^{a}$, S.D. SArkar ${ }^{a, b}$, B. Choudhury ${ }^{a}$ And A. Bhattacharjee ${ }^{a, *}$ \\ ${ }^{a}$ Department of Physics, NIT Silchar, Assam - 788010, India \\ ${ }^{b}$ Department of Physics, Karimganj College, Karimganj, Assam - 788710, India
}

(Received February 22, 2012; in final form August 10, 2012)

\begin{abstract}
The variation of refractive indices with temperature of three pure nematogenic compounds 5OCB, CPHB and 6CHBT were studied experimentally using thin prism technique. The refractive indices $n_{\mathrm{o}}$ and $n_{\mathrm{e}}$ were measured using $633 \mathrm{~nm}$ lines from a He-Ne laser. A modified four-parameter model was taken which is based on the Vuks equation describing the effect of temperature on the refractive indices of liquid crystals. In this paper we report the variation of refractive indices of these three liquid crystals with temperature, using the thin prism method. The variation in birefringence was determined experimentally. The variation in refractive indices and birefringence were also fitted theoretically using the modified Vuks equation. On comparison, the theoretically fitted values show close agreement with the experimental values.
\end{abstract}

PACS: 78.15.+e, 78.20.Fm, 64.70.M-

\section{Introduction}

The liquid crystalline (LC) state of matter is a fascinating subject from the point of both basic research and technological applications [1]. They possess many of the mechanical properties of liquid, e.g., high fluidity, formation and coalescence of droplets. At the same time they exhibit anisotropy in their optical, mechanical, electrical, and magnetic properties similar to that of crystals. This is why LCs attain their importance in technological applications, especially in electrooptics watches and in liquid-crystal displays (LCDs) [2]. At present the knowledge of such materials is more widespread thus increasing the use of liquid crystal devices in our everyday lives. LCDs are used in hand held phones, laptop screens and they are replacing the obsolete cathode ray tubes as television screens [3]. Among the many optical properties, the refractive indices of LCs play the most important role and their temperature dependence is of large importance from the application point of view [4,5]. Due to anisotropy of LCs, a properly aligned LC exhibits two refractive indices viz., ordinary $\left(n_{\mathrm{o}}\right)$ and extraordinary $\left(n_{\mathrm{e}}\right)$ refractive index. The difference of the two values gives the birefringence value i.e., $\Delta n=n_{\mathrm{e}}-n_{\mathrm{o}}$ and is an important factor in understanding the behavior of LCs. Most liquid crystal light modulators, e.g., flat panel display devices function by utilizing the electric-field-induced variation in refractive index. In addition to the molecular constituents, the operating wavelength and temperature are the most important factors in affecting the refractive indices of LCs [5-9]. Ordinary and extraordinary refractive indices of liquid crystals behave differently from each other as the temperature is increased.

Several experimental techniques have been developed

* corresponding author; e-mail: ayonbh@gmail.com to describe the temperature dependence of refractive indices of liquid crystals [10-25]. In this paper we have studied the optical properties of three nematogens by the measurement of their refractive indices as well as birefringence as a function of temperature using the thin prism method. We have considered a four-parameter model based on the Vuks equation describing the temperature effect of liquid crystal refractive indices and then we validate the model by fitting the experimental data of the three LC compounds. This model has been used by several authors for correlating theoretical values and experimental data of refractive indices of different LC compounds $[4-9,26]$. In this paper, we fitted the experimental data at different temperatures for three different LCs at an operating wavelength $\lambda=633 \mathrm{~nm}$, into the Vuks equation and found that the Vuks equation is being validated in our case.

\section{Experimental}

The LC compound 5OCB used in this work was procured from Frinton Labs, USA, the LC compound 6CHBT was obtained from Military University of Technology, Poland and the LC compound CPHB was obtained from North Bengal University, India.

Measurements of refractive indices were done by using the thin prism method with a He-Ne laser of wavelength $633 \mathrm{~nm}$ in a setup as shown in Fig. 1. Each sample was introduced into a wedge shaped glass prism of angle between $1^{\circ}-2^{\circ}$. In order to construct the prism, the glass slides whose inner surfaces were coated with $1 \%$ PVA solution have been used. Then the coated glass plates were rubbed by using a velvet cloth or tissue paper. Unidirectional rubbing was done to obtain fine and uniform grooves in order to promote the alignment of liquid crystals in the nematic phase. The prisms were then formed by sandwiching the two rubbed glass plates with a thin 
spacer at one end so that the angle of prism is within $1^{\circ}-2^{\circ}$. The LC samples were introduced into the prism in the liquid state through the open edge of the prism through capillary action. The prism encapsulated in a hot stage was kept on a rotating prism table where the temperature was controlled and monitored. The samples were heated to temperatures beyond the isotropic temperatures and then allowed to cool.

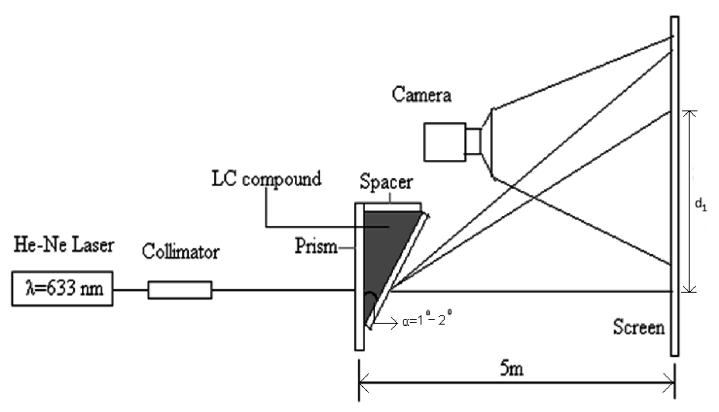

Fig. 1. Optical geometry used for the measurement of refractive index.

When the laser beam from the source was made incident on the sample through a hole drilled in the hot stage, the beam got split into two refracted spots owing to birefringence. The images of the spots were observed on a screen kept at a distance of $5 \mathrm{~m}$ from the prism and were photographed using a high resolution digital camera. The images of these spots were transferred to a computer and then further analyzed using software. Each sample was heated at the rate of $1^{\circ} \mathrm{C} / \mathrm{min}$ to a temperature beyond its isotropic temperature and allowed to cool at the same average rate. All the measurements were taken while cooling the sample from the isotropic state. The temperature of the prism encapsulated in the hot stage was regulated manually with an accuracy of $\pm 1^{\circ} \mathrm{C}$.

The ordinary $\left(n_{\mathrm{o}}\right)$ and extraordinary $\left(n_{\mathrm{e}}\right)$ refractive indices were calculated using the formula [10]:

$$
\begin{aligned}
& n_{\mathrm{o}}=\left(1+\frac{2 d_{2}^{\mathrm{o}}}{d_{1}}\right), \\
& n_{\mathrm{e}}=\left(1+\frac{2 d_{2}^{\mathrm{e}}}{d_{1}}\right),
\end{aligned}
$$

where $d_{1}$ is the distance between the positions of the image of direct transmitted beam on the screen from the image of the first reflected beam on the screen. $d_{2}^{\circ}$ and $d_{2}^{\mathrm{e}}$ are, respectively, distance of the ordinary spot and that of the extraordinary spot from the direct spots. $n_{\mathrm{o}}$ and $n_{\mathrm{e}}$ represent, respectively, the ordinary and extraordinary refractive indices. The accuracy of the system for measuring refractive indices is up to the fourth decimal.

\section{Theory}

Vuks proposed a semi-empirical model correlating the microscopic molecular polarizabilities to the macroscopic refractive indices of some crystalline materials. Vuks model has also been used to describe the wavelength and temperature dependences of the LC refractive indices. Though in the Vuks formula the local field is assumed to be isotropic, the application of this formula to liquid crystals leads to satisfactory results $[5-9,26]$.

The average refractive index of a LC is defined as

$$
\langle n\rangle \approx \frac{n_{\mathrm{e}}+2 n_{\mathrm{o}}}{3} \text {. }
$$

In Eq. (3) $\langle n\rangle$ represents the average refractive index of the LC sample.

As observed from our experimental data, the average refractive index $\langle n\rangle$ decreases linearly as the temperature increases. The fitted values of $\langle n\rangle$ also follows the same trend and is given by the equation

$$
\langle n\rangle=A-B T \text {. }
$$

Equation (4) has a negative slope and the value of $B$ is reported to be around $10^{-4} \mathrm{~K}^{-1}$ [9]. On the other hand, the birefringence is dependent on the order parameter $S$. Based on Haller's approximation, the order parameter can be approximated as $S=\left(1-T / T_{\mathrm{c}}\right)^{\beta}$. Thus, the temperature dependent birefringence has the following form [9]:

$$
\Delta n=\Delta n_{\mathrm{o}}\left(1-\frac{T}{T_{\mathrm{c}}}\right)^{\beta},
$$

where $\Delta n_{\mathrm{o}}$ is the LC birefringence in the crystalline state, the exponent $\beta$ is the material constant and $T_{\mathrm{c}}$ is the clearing temperature of the LC material under investigation.

In our studies, we have considered a four-parameter model [9] describing the temperature effect on the LC refractive indices which eventually gives the theoretical value of ordinary refractive index $n_{\mathrm{o}}$ and extraordinary refractive index $n_{\mathrm{e}}$ :

$$
\begin{aligned}
& n_{\mathrm{e}} \approx A-B T+\frac{2 \Delta n_{\mathrm{o}}}{3}\left(1-\frac{T}{T_{\mathrm{c}}}\right)^{\beta}, \\
& n_{\mathrm{o}} \approx A-B T-\frac{\Delta n_{\mathrm{o}}}{3}\left(1-\frac{T}{T_{\mathrm{c}}}\right)^{\beta} .
\end{aligned}
$$

We can see that Eqs. (6) and (7) have four fitting parameters viz., $A, B, \Delta n_{\mathrm{o}}$ and $\beta$. The parameters $A$ and $B$ can be obtained from fitting the temperature-dependent $\langle n\rangle$ parameters data at a given wavelength and parameters $\Delta n_{\mathrm{o}}$ and $\beta$ can be obtained by fitting the temperature-dependent $\Delta n$ data. Thus, once we have measured the temperature dependent $n_{\mathrm{o}}$ and $n_{\mathrm{e}}$, we rearrange the data into $\langle n\rangle$ and $\Delta n$ and parameters $A, B$, and $\Delta n_{\mathrm{o}}, \beta$ can be obtained directly.

To validate the four-parameter model, we use this model to fit the experimental data of three samples $5 \mathrm{OCB}, \mathrm{CPHB}$, and 6CHBT measured at $\lambda=633 \mathrm{~nm}$.

\section{Results and discussion}

\subsection{Experimental results}

The chemical names and the phase transition temperatures of the three LC compounds are as follows: 
1. 4-cyano-4-pentyloxy biphenyl (5OCB)

$\mathrm{Cr} \stackrel{53^{\circ} \mathrm{C}}{\longrightarrow} \mathrm{N} \stackrel{68^{\circ} \mathrm{C}}{\longrightarrow}$ Iso,

2. 4-heptyl-benzoic acid 4-cyano-phenyl ester (CPHB) $\mathrm{Cr} \stackrel{44.5^{\circ} \mathrm{C}}{\longrightarrow} \mathrm{N} \stackrel{57^{\circ} \mathrm{C}}{\longrightarrow}$ Iso,

3. 4(trans-4'-n-hexylcyclohexyl) isothiocyanatobenzene (6CHBT)

$$
\mathrm{Cr} \stackrel{1{ }^{\circ} \mathrm{C}}{\longrightarrow} \mathrm{N} \stackrel{42.8^{\circ} \mathrm{C}}{\longrightarrow} \text { Iso. }
$$

As the temperature is lowered from isotropic to nematic phase, the isotropic value splits into two, one value higher and another lower than the isotropic value corresponding to $n_{\mathrm{e}}$ and $n_{\mathrm{o}}$ refractive indices, respectively. It was found that $n_{\mathrm{o}}$ increases and $n_{\mathrm{e}}$ decreases as temperature was increased up to isotropic temperature. The values of $\Delta n$ and $\langle n\rangle$ are found to decrease as temperature was increased.

\subsection{Validation of the four-parameter model}

As previously discussed, the four-parameter model has four unknowns $A, B, \Delta n_{\mathrm{o}}$ and $\beta$. To obtain the values of $A, B, \Delta n_{\mathrm{o}}$ and $\beta$, iterative fitting was done in two steps. The values of refractive indices as a function of temperature were obtained experimentally. Once the refractive indices are measured, birefringence and average refractive indices can be calculated. In the first step of fitting we used Eq. (3) to fit the average refractive index data at different temperatures and obtain the parameters $A$ and $B$. In the second step we used Eq. (4) to fit the birefringence data and the parameters $\Delta n_{\mathrm{o}}$ and $\beta$ are obtained at different temperatures. Once these two sets of parameters are obtained, the values are used in Eqs. (6) and (7) to calculate the refractive indices $n_{\mathrm{o}}$ and $n_{\mathrm{e}}$. These fitted values are compared with the experimentally obtained ones. Three pure samples: 5OCB, CPHB, and 6CHBT were used to validate the four-parameter model. In our studies excellent agreement is obtained between the fitted values and experimentally measured data of all the three different samples. The fitting parameters for the three samples are listed in Table.

TABLE

Fitting parameters for 5OCB, CPHB, and $6 \mathrm{CHBT}$ at $633 \mathrm{~nm}$.

\begin{tabular}{c|c|c|c|c}
\hline \hline $\begin{array}{c}\text { Name of the } \\
\text { LC sample }\end{array}$ & $A$ & $B\left[\mathrm{~K}^{-1}\right]$ & $(\Delta n)_{\mathrm{o}}$ & $B$ \\
\hline 5 OCB & 1.7411 & $4.92 \times 10^{-4}$ & 0.3409 & 0.2125 \\
CPHB & 1.685 & $4.81 \times 10^{-4}$ & 0.243 & 0.1885 \\
$6 \mathrm{CHBT}$ & 1.71 & $4.95 \times 10^{-4}$ & 0.246 & 0.1897
\end{tabular}

Figure 2 shows the temperature dependent average refractive indices of the $\mathrm{LC}$ compounds (a) 5OCB, (b)

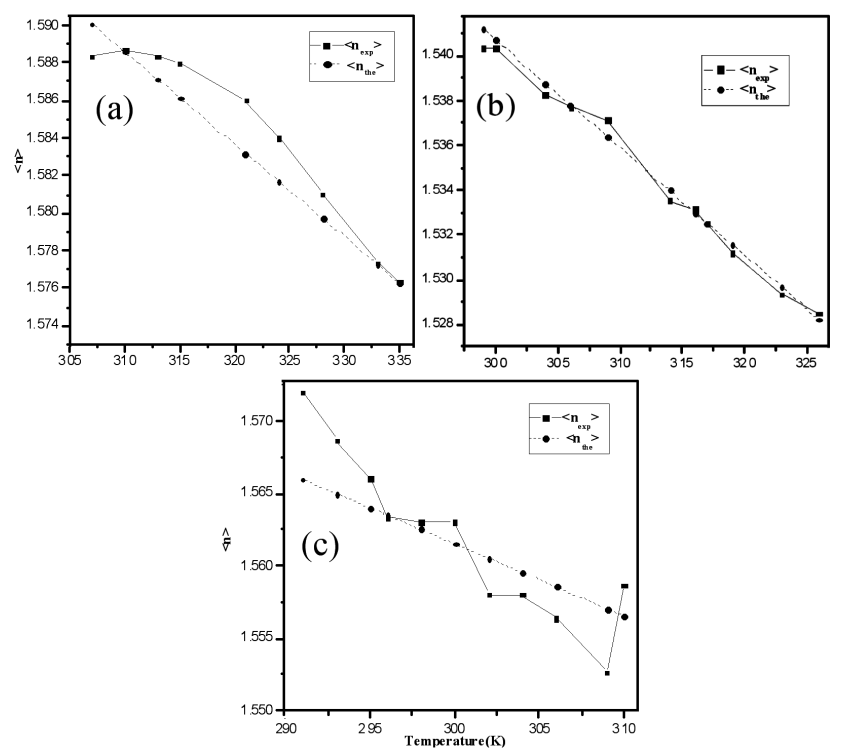

Fig. 2. Temperature variation of average refractive indices $\langle n\rangle$ of (a) 5OCB, (b) CPHB, (c) 6CHBT. The squares and circles respectively represent the experimentally measured values and the fitted values of $\langle n\rangle$ using Eq. (3). The fitting parameters are listed in Table.

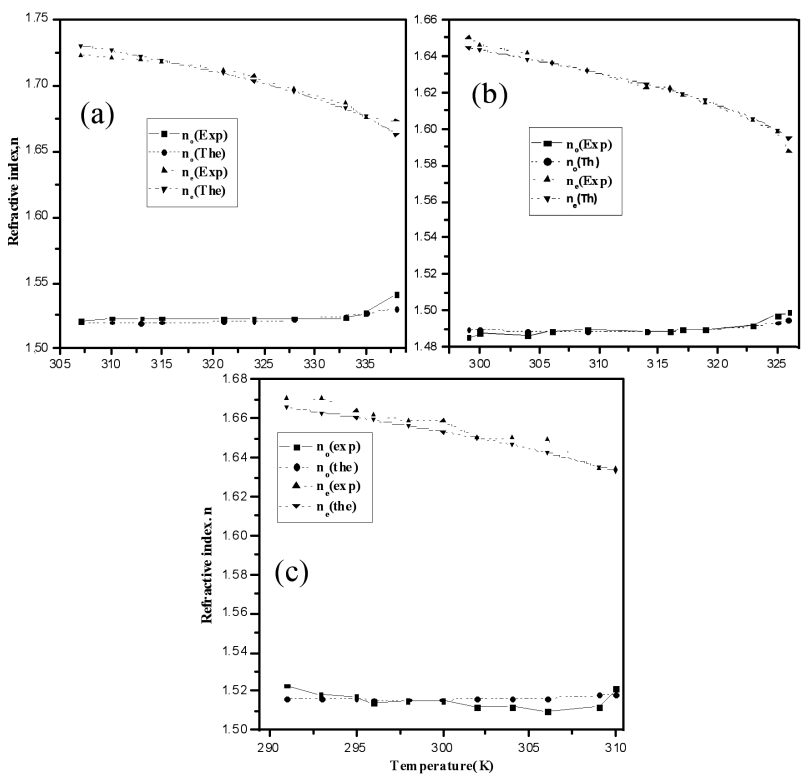

Fig. 3. Temperature variations of refractive indices of (a) 5OCB, (b) CPHB, (c) 6CHBT. Squares and upward-triangles represents experimentally measured values of $n$ while circles and downward triangles represent fitted values of $n$ using Eqs. (5) and (6). The fitting parameters are listed in Table.

CPHB, and (c) 6CHBT at a particular wavelength $\lambda=$ $633 \mathrm{~nm}$.

Figure 3 shows temperature variation of refractive indices of LC compounds (a) 5OCB, (b) CPHB, (c) 6CHBT at a particular wavelength $\lambda=633 \mathrm{~nm}$.

Likewise, Fig. 4 shows the temperature variation of average refractive indices of the LC compounds (a) 5OCB, 


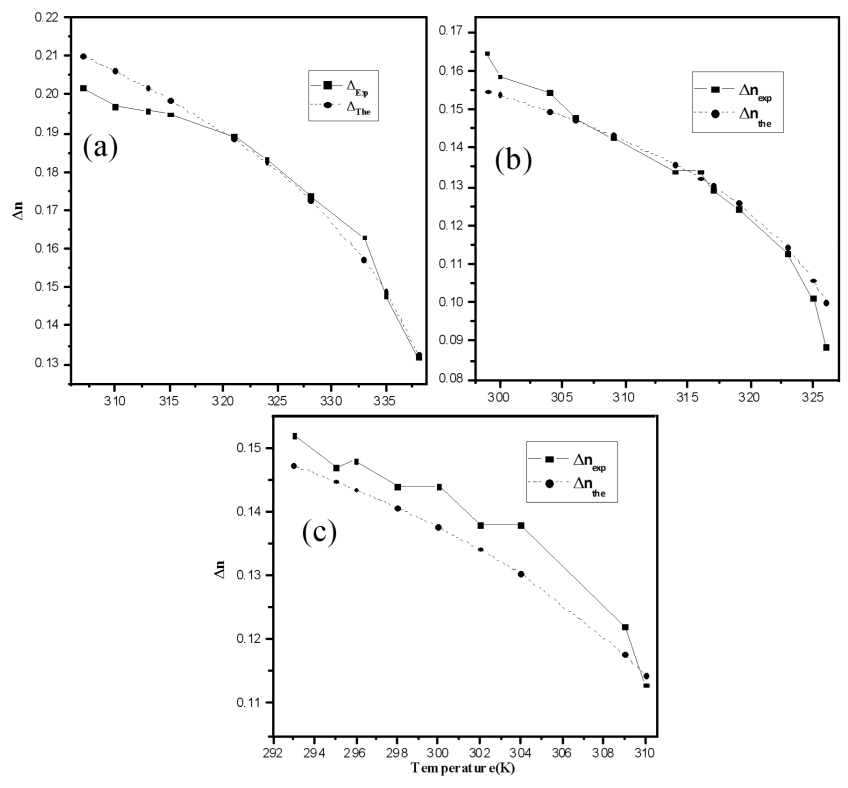

Fig. 4. Temperature variation of birefringence $(\Delta n)$ of (a) 5OCB, (b) CPHB, (c) 6CHBT. Squares and circles respectively represent the experimentally measured values and fitted values of $\Delta n$ using Eq. (4). The fitting parameters are listed in Table.

(b) CPHB, and (c) 6CHBT at a particular wavelength $\lambda=633 \mathrm{~nm}$.

As shown in the figures excellent agreement between the four-parameter model and experimental data is obtained.

\section{Conclusion}

The study of optical properties of three nematogens by the measurement of their refractive indices as a function of temperature and the variation of birefringence with different temperatures is presented in this paper. As expected, it was found that the refractive indices for extraordinary ray decreases with the increase of temperature and the refractive indices for ordinary rays are found to be increased with temperature. The birefringence $\Delta n=n_{\mathrm{e}}-n_{\mathrm{o}}$, exhibited by the compounds was observed to be higher at lower temperatures and somewhat diminished as the temperature increases. We have used a modified four-parameter model for describing the temperature effect on the refractive indices of liquid crystals based on the Vuks equation. Three different pure nematic LCs are used to validate this four parameter model. Excellent agreement between the experimental data and fitted values by using four-parameter model is obtained.

Finally, to summarize, the modified four-parameter model is found to be fit very well with all the different pure LC samples we studied. It can be assumed to be an accurate model to study the effects of temperature on the refractive index of liquid crystal materials.

\section{Acknowledgments}

The author would like to thank Prof. K. Czupryński, Military University of Technology, Poland for providing the sample 6CHBT and also Dr. M.K. Das, North Bengal University, for providing the sample CPHB used in the experiment.

\section{References}

[1] M. Roushdy, Liq. Cryst. 31, 371 (2004).

[2] M. Roushdy, Mol. Cryst. Liq. Cryst. 457, 151 (2006).

[3] R.A.M. Hikmet, Liq. Cryst. 33, 1407 (2006).

[4] J. Li, S.T. Wu, J. Appl. Phys. 95, 896 (2004).

[5] A.K. Singh, R. Manohar, J.P. Shukla, A.M. Biradar, Acta Phys. Pol. A 116, 485 (2006).

[6] J. Li, S.T. Wu, J. App. Phys. 95, 6253 (2004).

[7] A. Kumar, Acta Phys. Pol. A 112, 1213 (2007).

[8] T.N. Soorya, S. Gupta, A. Kumar, S. Jain, V.P. Arora, B. Bahadur, Ind. J. Pure Appl. Phys. 44, $524(2006)$.

[9] J. Li, S. Gauza, S.T. Wu, J. Appl. Phys. 96, 19 (2004).

[10] J.V. Yakhmi, V.K. Kelkar, R.P. Shukla, C. Manohari, Mol. Cryst. Liq. Cryst. 53, 55 (1979).

[11] D. Gupta, A. Mukhopadhyay, S.K. Roy, Mol. Cryst. Liq. Cryst. 506, 77 (2009).

[12] S. Brugioni, R. Meucci, S. Faetti, Mol. Cryst. Liq. Cryst. 465, 51 (2007).

[13] I.H. Ibrahim, W. Haase, J. Phys. (France) 40, 191 (1979).

[14] I. Palarie, C. Florea, J. Optoelectron. Adv. Mater. 7, 997 (2005).

[15] D. Sunanda, M.S. Madhava, D. Revannasiddaiah, R. Somashekar, J. Mahadeva, Mol. Cryst. Liq. Cryst. 409, 163 (2004).

[16] M.S. Sen, P. Brahma, S.K. Roy, D.K. Mukherjee, S.B. Roy, Mol. Cryst. Liq. Cryst. 100, 327 (1983).

[17] H.J. Muller, W. Haase, Mol. Cryst. Liq. Cryst. 409, 127 (2004).

[18] S. Chakraborty, A. Mukhopadhyay, S.K. Roy, Mol. Cryst. Liq. Cryst. 487, 13 (2008).

[19] S. Chakraborty, A. Mukhopadhyay, S.K. Roy, Mol. Cryst. Liq. Cryst. 493, 31 (2008).

[20] Y. Takanishi, M. Yoshiimoto, K. Ishigawa, H. Takezoe, Mol. Cryst. Liq. Cryst. 331, 619 (1999).

[21] K. Kali, S. Sen, S. Kumar Roy, Bull. Chem. Soc. Jpn. 58, 3576 (1985).

[22] S. Datta Sarkar, B. Choudhury, Acta Phys. Pol. A 118, 665 (2010).

[23] S. Datta Sarkar, B. Choudhury, Mol. Cryst. Liq. Cryst. 543, 214 (2011).

[24] S. Chakraborty, A. Mukhopadhyay, S.K. Roy, Mol. Cryst. Liq. Cryst. 482, 21 (2008).

[25] R.G. Horn, J. Phys. (France) 39, 105 (1978).

[26] J. Li, S. Gauza, S.T. Wu, Opt. Expr. 12, 4193 (2002). 